\title{
Trees of Tales: A Playful Reading Application for Arabic Children
}

\author{
Fatma Al Aamri, Stefan Greuter, Steffen P. Walz \\ GEElab, RMIT University \\ Melbourne, Australia \\ \{fatma.alaamri, Stefan.greuter, steffen.walz\}@ermit.edu.au
}

\begin{abstract}
In this study, we have developed a playful interactive reading and storytellng application called 'Trees of Tales'. The tablet application was designed to motivate Arabic children to read more for pleasure. 'Trees of Tales' was evaluated with 18 primary school children in Oman to investigate its effectiveness as an enjoyable reading tool. To assess the impact on usability and the children's experience of fun and reading preference, we conducted three reading sessions in which all participants read stories from 'Trees of Tales', non-interactive e-books and conventional printed books. This paper describes the design of the 'Trees of Tales' application as well as the results obtained with the 'fun toolkit'.
\end{abstract}

Keywords. Interactive Reading Application, Trees of Tales, Children e-books, Arabic children, Reading for pleasure.

\section{Introduction}

Islamic cultures, by the way of the Quran, emphasise that reading is quintessential to humankind. The first word of Quran sent to the Prophet Mohamed was the word 'Read', and it was repeated three times emphasising its importance (Quran, 96.1). Reading at home contributes to children's concentration abilities, social collaboration, and language development [2]. However, the huge decline in reading and publishing in the Arab world has been reported. The Arab Thought Foundation Fikr released in its fourth annual cultural development report in January 2012 that Arabic children read on average six minutes a year compared to the approximately 12,000 minutes western children are engaged in reading per year [1]. Arabic children often only read their textbooks and the Holy Quran as part of their educational and religious needs. However, they do not read for pleasure as much as wester people read despite the importance of reading that the Quran itself underlines.

Some Arabic countries such as the Gulf countries are often classified as highincome countries and often have a high literacy rates. Oman is listed within the best performing 35 countries in the world [21]. According to UNICEF statistics, literacy rates of children aged 15 years and older reached $97.4 \%$ for males and $98.2 \%$ for females in 2012 [22]. Literacy is therefore not the problem that is leading to the decline in reading. 
Advanced technology is widely available in Gulf countries. In 2013, 181 mobile phones and 60 Internet users were registered for every 100 people in the area's population [22]. Despite the prevalence of technology however, there is limited access to reading materials for Arabic children. Particularly interactive e-books and apps in Arabic language are very rare.

Recent research indicated that interactive digital books provide children with enjoyment through interactivity, which motivates them to continue reading [10]. Animation in interactive storybooks was also linked to improved information recall [19]. However, as far as we know, there has not been any research on the effectiveness of interactive reading applications to motivate Arabic children to read. This paper compares and describes the experiences and opinions of Arabic children who read as part of three interventions that we compared: (a) an interactive reading application 'Trees of Tales', (b) a non-interactive e-book application 'Arabic Stories', and (c) traditional print books available in Omani Primary school libraries.

\section{$2 \quad$ Reading for Pleasure}

Research indicates that children, who enjoy reading, read more frequently and their reading proficiency increases. Several benefits result from reading for pleasure activities [6]. They include elevated levels of reading attainment, writing ability, text comprehension, grammar, breadth of vocabulary, positive reading attitudes, greater selfconfidence, and pleasure reading in later life. The huge benefit of reading drives governments and organisations to encourage their people to read more [6].

Independent reading for pleasure is the most important indicator of the future career and social achievements of a child [11]. However, without motivation it is difficult to engage even the brightest children in reading or any other learning activities [15]. It was found that intrinsic motivation predicts an increase in the amount of reading for pleasure more than extrinsic motivation [7], [23]. Interest and challenge are the two main components of intrinsic reading motivation [9]. Therefore, it is important to foster the interest and curiosity of children through providing a range of reading topics that suits their ages and cultures.

\section{Interactive E-books}

E-book publishers and designers have attempted different approaches to use interactivity in e-books to motivate and engage children as well as increase their comprehension of the content [16]. Thus, research on how interactivity is used in e-books and how it can be used in an engaging and effective manner is important. Children need to be moved away from interactive e-books that merely entertain towards e-books that educate, whilst they entertain.

Most of the interactivity that occurs in e-books exists to improve the engagement of children with the application. Such applications are enjoyable and attractive for children and motivate them to continue interacting with the application. Recent evidence, however, suggests that children who read e-books with many interactive ele- 
ments recalled significantly less narrative details than children who read print versions of the same books [5]. Conversely, Labbo and Kuhn [12] found that e-books, which integrated corresponding interactivity into the content of the story, were found to elevate the comprehension of children.

Additionally, it was found that features such as narration, sound effects and animation, which support the text, help to remove the effort from decoding individual words and allow children to focus on meaning [13, 14]. Digital books are known for their non-linear storytelling potential, which means "the telling of a story with an audience impact on the storyline, but not on the story goal or the end of the story" [3]. Therefore, one design approach to tap into this potential is to allow for the reader to intervene within storylines, and to try and match the content of the story to the visual elements. These audience-based actions may be resources for enjoyment and engagement and a method to reinforce the understanding of the story.

\subsection{A Reading Application for Arabic Children (Trees of Tales)}

The design of the reading application 'Trees of Tales' included Arabic traditional folktales in order to elevate the interest of Arabic children in the reading. Game elements are added to the application to provide playful experiences that encourage children to continue with the reading. Additionally, to provide flexibility of place and time for reading, we decided to use the iPad as a platform for the application.

'Trees of Tales' aims to provide Arabic children aged eight and above with a collection of interactive stories that motivate them to read for pleasure and that are based in their culture. The application also provides children with the option of creating new stories using the characters and backgrounds that are available in the existing stories. It includes interactivity such as dragging and dropping objects to the scene that aimed to keep children engaged. One of the main design considerations in Trees of Tales was to ensure that children were actually reading the stories while engaging with the interactive features available. To this end, children are asked to set the scene and manage a few actions in the story such as selecting and positioning the relevant pose of the character on the scene and adjusting the emotional state of the characters in correspondence with the text. Only if the scene is set in accordance with the text, the next page of the story will unlock.

Feedback was also provided to children when their choice of actions was wrong. For example, a selected character would not stick on the background and return to its previous position if moved to a wrong position. Similarly, when children looked for the hiding thieves in Joha's first story, a sad sound tune assisted children to recognise a wrong position. Ultimately, when the scene was completed according to the text, the Next Button appeared green and a jingle indicated that the child could proceed to the next page. Similar design elements and the Next Button existed on every page. Screen shots of a story page of 'Trees of Tales' showing the interactions are illustrated in Figure 1. If children changed a critical element that made the scene contradict with the storyline, the green button turned back to red again to indicate that there was something incorrect in the scene and the next page was locked again. However, chil- 
dren were able to be creative by adding other images or re-arranging the scene in a way that did not affect the storyline.
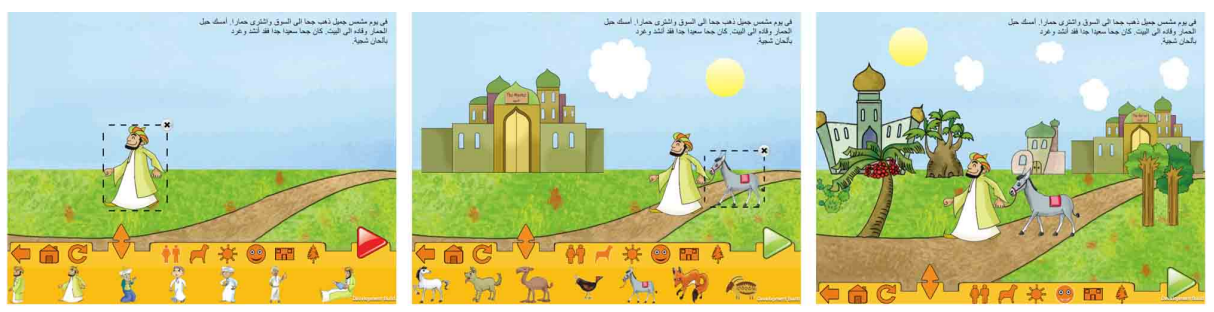

Fig. 1. Screen shots of a scene in 'Trees of Tales'

\section{$4 \quad$ Experiment Design}

This study evaluated 'Trees of Tales' (TT) as an enjoyable reading experience in comparison to two other reading interventions; non-interactive application (EB), and printed storybooks (PB). For the non-interactive application, we chose an iPad app named 'Arabic Stories' that we had found in the App store and that contained five Arabic stories. The printed books were 40 storybooks selected from the school library with the help of the librarian and children chose 3 to 4 of the books to read. Previous studies of children experiences with educational software found that both 'observed' and 'reported' fun are informative in user studies (Read, MacFarlane, \& Casey, 2002). In this paper, we concentrated on the 'reported' fun, usability, and preference for reading.

An experiment of within-subjects counter-balanced design with the three interventions: TT, EB, and PB was performed. The sample comprised 18 children ( 9 boys, 9 girls) aged between 9 years and 10 years. The children were randomly selected from fourth grade of a public primary school in Muscat, Oman. To determine the order in which children used the three interventions, a counter-balanced design was applied (Foley, 2004). This approach required the division of the sample into six groups that read from the three interventions in different order.

The experiment was carried out over three weeks. One reading session was performed every week during the school's 25-minute break and in the library room. On the second and third weeks, the same children were allocated to a different reading intervention. Over the course of the three weeks, every child used each of the three interventions once.

\subsection{Data Collection}

The primary means of data collection was the 'fun sorter', which is part of the 'fun toolkit' [18]. The 'Fun Toolkit' is a data collection tool that assesses the opinions of children and their reaction to software, applications or games [20]. The 'fun sorter' required the children to rank the three reading interventions in order of preference on three separate criteria: (a) fun, (b) ease of use, and (c) how interesting the content 
was. The method provided each child with a form containing three spaces for each question and 'stickers' depicting the interventions. The pictures on the stickers were explained to them. The children were then asked to rank the three interventions by sticking the three stickers into the spaces on the form in the appropriate order. A question in the bottom of the form asked which intervention the children would choose to read again. For this particular question, the children could only choose one sticker to answer this question. All of the children completed the 'fun sorter' questionnaire and, from our observation, seemed to enjoy the activity (Fig. 2).

The 'fun sorter' instrument provided insight into the reading intervention the children enjoyed the most, the reading intervention children found the easiest to use and the reading intervention that contained the most interesting stories. Additionally, we found out which reading intervention children would select to read again.
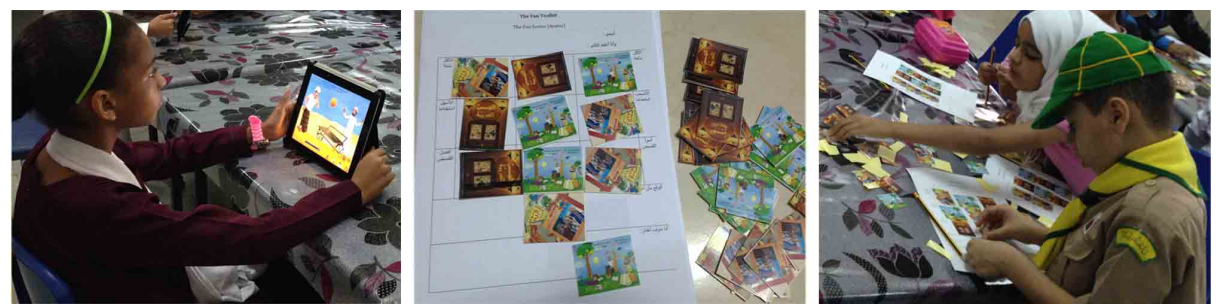

Fig. 2. Images from the Experiment

\section{$5 \quad$ Results and Discussions}

The Fun Sorters completed by the children were coded in an ordinal manner 1-3 for each of the criteria Fun, Ease of use, and Best content. For example, 3 represented most fun and 1 least fun. The last question in the 'fun sorter' (I would choose to read from) was scored according to how many children chose that intervention. Table 1 shows, for each criterion, how many children ranked each intervention highest.

Table 1. Frequency each intervention was ranked first in the fun sorter.

\begin{tabular}{|l|c|c|c|}
\hline & TT & EB & PB \\
\hline Fun & $\mathbf{1 1}$ & 7 & 0 \\
\hline Ease of use & 2 & $\mathbf{1 1}$ & 5 \\
\hline Best content & $\mathbf{1 2}$ & 6 & 0 \\
\hline Child choice & $\mathbf{1 3}$ & 5 & 0 \\
\hline
\end{tabular}

The mean scores for fun were $\mathrm{TT}=2.61, \mathrm{~EB}=2.27, \mathrm{~PB}=1.11$. A Friedman test revealed statistical significant difference in perceived fun on the different reading interventions, $\chi^{2}=22.33, p<0.0005$. Post hoc Wilcoxon tests revealed the PB were ranked significantly lower than both TT and EB, but the difference between the EB and the TT was not significant. Children found 'Trees of Tales' the most enjoyable reading experience of the three interventions tested. This could be due to the visual and interactive elements in the application that encourages children to build the scene of the stories in a playful sense. 
The mean scores for ease of use were $\mathrm{TT}=1.83, \mathrm{~EB}=2.39, \mathrm{~PB}=1.78$. A Friedman test revealed that there was no significant difference in perceived ease of use among the three interventions. However, table. 1 illustrates that only two children picked TT as the easiest to use, which indicates that the majority of children found TT not as easy to use as the other interventions. More than $50 \%$ of the children picked the non-interactive app as the easiest to use. This was expected as the application was direct and did not have interactivity that children had to complete except for picking a story and pressing next in each page.

The mean scores for best content were $\mathrm{TT}=2.67, \mathrm{~EB}=2.22, \mathrm{~PB}=1.22$. A Friedman test revealed statistical significant difference in perceived best content on the different reading interventions, $\chi^{2}=20.81, \mathrm{p}<0.0005$. Post hoc Wilcoxon tests revealed the TT was ranked significantly higher on best content than $\mathrm{PB}$, but the difference between EB and TT was not significant. This finding indicated that children found the stories in 'Trees of Tales' more interesting than the stories in the printed books. It is noteworthy to point here that the stories in 'Trees of Tales' were carefully selected from traditional folktales in Oman whereas the printed books were selected from the school library. Hence, providing children with stories and characters that relate to their culture, made it more interesting for them to read. This finding supports the view that interest is a factor of intrinsic motivation for reading [9]. School libraries should also consider providing interesting reading material to motivate children to read more.

The mean scores for children choice for each of the interventions were $\mathrm{TT}=0.72$, $\mathrm{EB}=0.27, \mathrm{PP}=0.00$. A Friedman test revealed that there was a statistical significant difference in children choices of the interventions to read. Post hoc Wilcoxon tests revealed that the significant difference was between the PB and TT. The difference in children choices between the PB and EB and between the EB and the TT was not significant. Interestingly most children in the sample preferred to read from the 'Trees of Tales' app although it was not easier to reading from. However, more research is needed to find out what aspect of 'Trees of Tales' children liked the most and what the most interesting features of the stories were. More information about the difficulties the children encountered while using 'Trees of Tales' would be interesting to inform the future development of the application.

The results presented here reveal that the decision whether or not to use an interactive reading application is based on how much fun it is perceived to be. Children's own reports of how much fun the interventions were to use and the most interesting content they found were correlated to their choices of the reading interventions they would like to continue using. However, their perceived ease of use did not correlate with their choice of intervention to read again. This could be due to their preference for challenge at this age and calls for further research. Challenge is one of the intrinsic motivation factors that influence reading for pleasure [9]. However, it is important for the application designers to balance challenge with abilities if we want children to continue reading in the future. 


\section{Conclusion}

This study investigated the effectiveness of designing and developing an interactive reading application specifically for Arabic children. 'Trees of Tales' was designed with the purpose motivating Arabic children to read for pleasure. The 'fun sorter' was used to canvas the opinions of the 18 children regarding the different reading interventions including 'Trees of Tales'. Participants indicated that they enjoyed reading from 'Trees of Tales' more than the e-book or the printed books. They revealed that the stories included in 'Trees of Tales' were more interesting than the stories in the printed books. However, the children found 'Trees of Tales' the most difficult to use out of the three interventions. The preliminary results indicate that 'Trees of Tales' has a positive impact on Arabic children's reading behaviour.

Overall, this experiment shows that using technology such as tablet PCs for pleasurable reading offered children more enjoyment than the existing reading media in schools such as printed books. Although there was no significant difference in perceived fun and best content between the non-interactive e-book stories and 'Trees of Tales', the children indicated that they would read 'Trees of Tales' again, which supported our suspicion that interactive media has an advantage over the static media in this case.

Obviously, the analysis of children 'reported' experiences, needs to be connected with the 'observed' situations to complete the picture and develop a conclusion on the effectiveness of 'Trees of Tales' [18]. Additionally, the collected data raised questions about the specific features of 'Trees of Tales' that children enjoyed the most or found difficult, which calls for a further investigation of the video recordings that were conducted during the experiment. Future work will include analysis of the observational data collected in the reading sessions.

\section{$7 \quad$ References}

1. Al-Yacoub, Ikram: (2012). Sum of All Fears: Arabs read an average of 6 pages a year, study reveals. Retrieved from http://english.alarabiya.net/articles/2012/07/14/226290.html.

2. Beauchat, K. A., Blamey, K. L., \& Walpole, S.: Building preschool children's language and literacy one storybook at a time. Reading Teacher, 63, 26-39 (2009)

3. Blythe, M.A., Monk, A.F., Overbeeke, K., \& Wright, P.C.: Funology: From Usability to Enjoyment: Kluwer Academic Publishers (2006)

4. Breakwell, G. M., Hammond, S., \& Fife-Schaw, C.: Research methods in psychology. London: SAGE (2000)

5. Chiong, C., Ree, J., Takeuchi, L., \& Erickson.: Print Books vs. E-books: Comparing parent-child co-reading on print, basic, and enhanced e-book platforms. The Joan Ganz Cooney Center at Sesame Workshop, New York (2012)

6. Clark, C., \& Rumbold, K.: Reading for Pleasure: A research overview. London. National Literacy Trust, 30 (2006)

7. Cox, K.E., \& Guthrie, J.T.: Motivational and Cognitive Contributions to Students' amount of Reading. Contemporary Educational Psychology, vol. 26, 116-131. (2001)

8. Foley, H.: Counterbalancing. In Michael S. Lewis-Beck, A. Bryman, \& Tim Futing Liao (Eds.), The SAGE Encyclopedia of Social Science Research Methods. (pp. 206-207). 
Thousand Oaks, Sage Publications, doi: http://dx.doi.org/10.4135/9781412950589.n180 (2004)

9. Guthrie, J. T. and Wigfield, A.: Engagement and motivation in reading. In M.L. Kamil, P.B. Mosenthal, P. D. Pearson, and R. Barr (Eds.), Handbook of Reading Research (3rd Ed.). New York: Longman (2000)

10. Hsu, M. C., \& Chen, C. P.: Analysis of Motivation Triggers in Interactive Digital Reading for Children. International Journal for Infonomics (IJI), Volume 6, Issues 1/2 (2013)

11. Kirsch, I., Jong, J. de., Lafontaine, D., McQueen, J., Mendelovits, J., \& Monseur, C.: Reading For Change: Performance and Engagement Across Countries. Organisation for Economic Co-operation and Development (OECD) (2002)

12. Labbo, L.D., \& Kuhn, M.R.: Weaving chains of affect and cognition: A young child's understanding of CD-ROM talking books. Journal of Literacy Research, 32 (2000), pp. 187$210(2000)$

13. Lewin, C.: Exploring the effects of talking book software in UK primary classrooms. Journal of Research in Reading, 23, 2, 149-157 (2000).

14. Matthew, K. A.: comparison of the influence of interactive CD-ROM storybooks and traditional print storybooks on reading comprehension. Journal of Research on Computing in Education, 29, 3, 263-275 (1997)

15. Metsala, J. L., Wigfield, A., Dacey, McCann A.: Children's Motivations for Reading. The Reading Teacher, 50(4), 3 (1996)

16. Pearman, C. J.: Independent reading of CD-ROM storybooks: Measuring comprehension with oral retellings. Reading Teacher, 61(8), 594-602 (2008)

17. Read, J. C.: Validating the Fun Toolkit: an instrument for measuring children's opinions of technology. Cognition, Technology \& Work, 10(2), 119-128 (2008)

18. Read, J. C., MacFarlane, S. J., Casey, C.: Endurability, engagement and expectations: Measuring children's fun. In Interaction Design and Children (Vol. 2, pp. 1-23). Eindhoven: Shaker Publishing (2002)

19. Seyit, E. I.: The impact of interactive storybook on elementary school students' recall. USChina Education Review, 140-146 (2011)

20. Sim, G., MacFarlane, S., \& Read, J.: All work and no play: Measuring fun, usability, and learning in software for children. Computers \& Education, 46(3), 235-248 (2006)

21. The World Bank. World Development Indicators (WDI). Data by country. Retrieved from http://data.worldbank.org/country/oman (2011)

22. UNICEF. (2013). Country statistics. Accessed on 20 January 2014. Retrieved from $\mathrm{http}: / /$ www.unicef.org/infobycountry/oman_statistics.html

23. Wang, J.H.Y. and Guthrie, J.T.: Modeling the effects of intrinsic motivation, extrinsic motivation, amount of reading, and past reading achievement on text comprehension between U.S. and Chinese students. Reading Research Quarterly, 39, 162-186 (2004)

24. Wigfield, A., Guthrie, J. T., Tonks, S., \& Perencevich, K.: Children's Motivation for Reading: Domain Specificity and Instructional Influences. The Journal of Educational Research, 97(6), 299-309 (2004) 